\title{
SIZE EVOLUTION OF THE LIGHT INDUCED SODIUM CLUSTERS*
}

\author{
B. Hnat, D. Jakubczyk, K. Kolwas and M. Kolwas \\ Institute of Physics, Polish Academy of Sciences \\ Al. Lotników 32/46, 02-668 Warsaw, Poland
}

(Received May 5, 1992)

\begin{abstract}
We report the observation of the size evolution of the laser light formed clusters. A cell containing sodium vapor and the buffer gas (He) under high pressure was used in the experiment. Changes of the radius and of the density of transient clusters were observed via the time evolution of the intensity of right-angle scattered light. The rate of the cluster radius growth was found to vary from 0.012 to $0.1 \mu \mathrm{m} / \mathrm{s}$.
\end{abstract}

PACS numbers: $36.40 .+\mathrm{d}, 31.70 . I I q, 64.70 .-\mathrm{p}$

\section{Introduction}

Since first clusters were observed in physical experiments a lot of methods have been established to enrich our knowledge about their main features. Considerable interest has been shown in the study of formation and structure of clusters [1-7]. Special attention has been paid also to the phase transitions of clusters according to the idea that the structure of cluster is also important for its stability $[8,9]$. A number of experiments have been focused on the problem of cluster properties such as ionization potentials, crystallographic structure, binding energy, chemical reactivity, and its dependence on the particle size known as the "quantum size" effect [10-13]. It has been shown that the occurrence of the "magic numbers" - the stability of certain cluster sizes - correspond to the spherical shell closings of the electronic energy levels $[2,13,14]$.

Great effort has been devoted to the production and study of ultrafine particles. There are several methods utilized for this purpose, e.g., using inert gases for cooling the alkali vapor to form small particles - let us mention only the cluster production by the seeded beam expansion [15] or the production of particles in a flowing inert gas (e.g., [16]).

*This work was supported by the State Commitee for Scientific Research Grant No. 204859101. 
In Ref. [17] the ultrafine particles of potassium were created by resonant laser excitation of potassium molecules contained in a heat-pipe oven. The mechanism of the process was similar to that from the experiment with inert gases - the vapor expansion was induced by laser light local heating. The heating caused the pressure/temperature imbalance and the expansion of the metal vapor into the colder buffer gas zones.

The studies of the interaction of clusters with the light leads to excitation spectra, light scattering patterns, and absorption coefficients, as well as to the nonlinear optical phenomena. This is the way to determine cluster properties [18-22]. Among others, the cluster size can be determined by means of analysis of the scattered light intensity pattern. What is more, it is non-destructive method. The phenomenon of the light scattering is used in a wide variety of methods: from simple sizing of the particle to the more complicated ones applied for phase transitions phenomena.

In this paper we use dynamic measurements of the light scattering to observe how the sodium clusters grow, after initialization by sudden switching on the laser light. In the reported experiment the sodium cell was illuminated with the blue $488 \mathrm{~nm}$ laser light of the Ar laser, resonant in respect to the well-defined transition in the rovibronic structure of sodium dimers. The laser light excited the well-known dimer fluorescence, e.g. [23], which could be observed through the side windows of the cell; the excited, fluorescing dimers formed a green cylinder with the base equal to the cross-section of the laser beam. Switching on the laser light was followed by the spectacular phenomena: after few seconds time, the volume of the green cylinder started to fill up with a dense, blue $(488 \mathrm{~nm}) \mathrm{fog}$, and the blue color became dominant. During the next few seconds the blue fog condensed into well-separated aggregates, appearing as the blue $(488 \mathrm{~nm})$ metallic reflections on the green background. The aggregates performed Brownian motions.

There are several phenomena which could initialize the condensation process, e.g.:

- the laser ionization of the sodium dimers;

- the resonant absorption of the light and local heating of the medium leading to the pressure and density gradients, and to condensation;

- the pushing of the dimers by the resonant laser light, leading to supersaturation of the vapor.

\section{Scattering of light}

The problem of the scattering of light by a conducting sphere was completely solved by Mie in 1908 [24]. The Mie solution determines the field in a given point inside or outside the sphere by means of Bessel functions and Legendre polynomials. Nevertheless, this solution is too complicated as far as the scattered light intensity as a function of both the size and the number of clusters is considered. Since our first estimations set the size of clusters close to the length of the light wave, we could apply neither a solution proper for very small nor for large particles. After many attempts we decided to use a simple Rayleigh-Gans scattering 
theory [25]. Thus, the scattered light intensity $i$ as a function of the cluster radius $r$ and the wavelength $\lambda$ is given by the formula

$$
i(x, \theta)=\frac{k^{4} V^{2}|m-1|^{2}}{8 \pi^{2} R^{2}} I_{0} G^{2}[2 x \sin (1 / 2 \theta)]\left(1+\cos ^{2} \theta\right),
$$

where $x=k R, k=2 \pi / \lambda, G(u)$ is proportional to Bessel function $G(u)=$ $\left(9 \pi / 2 u^{3}\right)^{1 / 2} J_{3 / 2}(u), \theta$ is the scattering angle, and $R$ is the distance from the particle. $m=m_{1} / m_{2}$, where $m_{1}$ and $m_{2}$ are the refractive indices of the sphere and of the medium, respectively. In our experiment we used helium as a buffer gas and therefore the refractive index is close to 1 and we can put $m=m_{1}$.

The intensity of the light scattered in the direction defined by an angle $\theta$ by a growing particle exhibits a train of maxima and minima. This train resembles the Bessel function. It is possible, in principle, to determine the particle size by counting the minima. It can be done as well by determining the frequency of the oscillations of the intensity. We get it automatically by fitting the theoretical curve to the experimental data. Thus, we need a formula describing the phenomenon.

The Bessel function can be approximated by cosine for the particles which are very large in respect to the wavelength. Therefore, for $\theta=90^{\circ}$ we obtain

$$
i\left(r, \theta=90^{\circ}\right)=\frac{r^{2}|m-1|^{2}}{32 R^{2}} I_{0} \cos ^{2}(\sqrt{2} k r) \text {. }
$$

We remember that this Eq. (2) describes the intensity of the light scattered by a single particle. If we neglect the multiple scattering, the total scattered light intensity is proportional to the Fromhold integral of product of the number of clusters of a given radius $N(r)$ and the one-particle scattering function

$$
I=\int N(r, t) i(r, \theta) \mathrm{d} r .
$$

The number of particles is a function of two variables: particle size and time. In fact the size of clusters is also a function of time, and the form of $N(r, t)$ and $I(r, t)$ is very complicated.

The most essential assumption, in a way derived from our microscope observation, is that the clusters' radius or at least their mean radius is well-defined and grows linearly in time until thermodynamic equilibrium is reached. This assumption is in agreement with the thermodynamic model of the growth of droplets in the supersaturated vapor [26].

We can further simplify $N(r, t)$ remembering that we are working under the conditions of relatively large $r$, i.e., the clusters are built of a great number of molecules (see the next section). Thus, we can assume that $r$ changes very slowly in time, so we can replace $N(r, t)$ by the product of two functions: $N=n(r) n(t)$. Then we assume that $n(t)$ behaves like a harmonic, dumped oscillator. This idea is supported by a popular model of two generations, in our case small and large clusters and a store of food ( $\mathrm{Na}_{2}$ dimers):

$$
n(t)=A \mathrm{e}^{-\Gamma t}[-\cos (\omega t)+C \sin (\omega t)]+1 \text {. }
$$

We just guess the shape of $n(r)$ in a form as simple as possible. Our aim is to obtain the decrease in the number of clusters with the increase in the radius

$$
{ }_{i}(r)=\frac{\text { const }}{r^{2}} \text {. }
$$


This expression simplifies our numerical model leading to an expression which can be understood phenomenologically

$$
N(r, t)=A \frac{\mathrm{e}^{-\Gamma t}[-\cos (\omega t)+C \sin (\omega t)]+1}{r^{2}} .
$$
tensity:

In the end one can obtain the following formula for the scattered light in-

$$
I(t)=A\left\{\mathrm{e}^{-\Gamma t}[-\cos (\omega t)+C \sin (\omega t)]+1\right\}[1+D \cos (\Omega t)]
$$

where $\Omega t=2 \sqrt{2} k r . A, \Gamma, D, \omega, \Omega$ are used as free parameters coming from the experiment. The parameter $D$ can be related with the fact that a diffusion of $r$ values around the mean value can exist. This parameter describes the legibility of the interference pattern created. For the extremal conditions it was necessary to put the exponential decay of the legibility, i.e., $D=d \mathrm{e}^{-\gamma t}$.

\section{Experiment}

The sodium vapor was contained in a temperature controlled cross-like heat-pipe oven. The beam from a single-mode argon-ion laser working on the $488 \mathrm{~nm}$ line was illuminating the vapor. It excited sodium dimers from their $X^{1} \Sigma_{\mathrm{g}}^{+}(v=3, j=43)$ level of the electronic ground state to the $(v=6, j=43)$ level of the $B^{1} \Pi_{\mathrm{u}}$ state. The laser induced phenomena were observed in the direction perpendicular to the laser beam (see Fig. 1) with the use of an interference

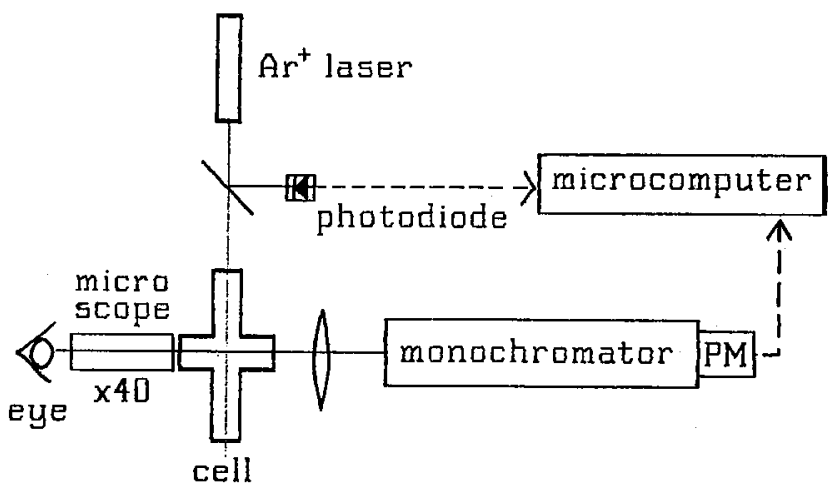

Fig. 1. Experimental arrangement.

filter with maximum at $488 \mathrm{~nm}$ or a monochromator and a photomultiplier. We could also observe the phenomenon through a 40 times magnifying microscope. The monochromator was set at the laser light wavelength $(488 \mathrm{~nm})$. The signal from the photomultiplier, proportional to the intensity of the laser light scattered by sodium clusters, was digitized. It was recorded as a function of time with the use of a microcomputer. The data was collected every $0.1 \mathrm{~s}$. The transient signals were registered for the parameters varying in a large range - temperature from $400^{\circ} \mathrm{C}$ up to $600^{\circ} \mathrm{C}$, He pressure from 300 to $700 \mathrm{Tr}$ and the laser power from 50 to $500 \mathrm{~mW}$. 
In this paper we would like to present the analysis of the cluster growth for two sequences of measurements:

- the dependence on the temperature for constant buffer gas pressure of $500 \mathrm{Tr}$ and the laser power of $300 \mathrm{~mW}$,

- the dependence on the buffer gas pressure for constant temperature of $463^{\circ} \mathrm{C}$ and the laser power of $150 \mathrm{~mW}$.

In Fig. 2 we show several examples of the experimental data - the intensity $I$ of the scattered light as a function of time, together with results of the numerical fit of the expression (7). One can notice that the train of the maxima and minima is superimposed on a slowly evolving maximum. This interference pattern is especially well pronounced for higher temperatures.

Fitting of the Eq. (7) to the experimental data makes possible to find the frequency $\Omega$ and by this means the rate of growth of the particles $v$, which is shown in Fig. 3. For the temperatures below $500^{\circ} \mathrm{C}$ the rate of cluster growth is almost constant and equal to $c a .0 .016 \mu \mathrm{m} / \mathrm{s}( \pm 0.002)$. For higher temperatures it increases rapidly reaching the value of $0.1 \mu \mathrm{m} / \mathrm{s}$ for $529^{\circ} \mathrm{C}$. We would like to stress that thanks to the richness of the temporal structure of the scattered light intensity $I$ the frequency $\Omega$ describing the rate of cluster growth is very well-defined. This rate of growth of clusters (in e.g. micrometers per second) was calculated from the equation $\Omega t=\sqrt{2} k r$ and is equal to

$$
v=\frac{r}{t}=\Omega \frac{\lambda}{4 \sqrt{2} \pi},
$$

with $\lambda=0.488 \mu \mathrm{m}$.

The dependence of the evolution of clusters on the buffer gas pressure for the constant temperature is shown on the sequence of Fig. 4.

In this case the evolution of the scattered light intensity was measured with a small constant and with no averaging of data, to visualize the fluctuations of intensity. The dispersion of the experimental data points is connected with fluctuations of the scattered light intensity rather than with the noise introduced by the equipment. In this case we present the data without any smoothing to show the fluctuations as well as the quality of our phenomenological fits. But we will not discuss here the problem of the fluctuations around the mean value, approximated by means of Eq. (6).

For the highest values of pressure (700 and $675 \mathrm{Tr}$ ) the evolution of the observed intensity is just the continuous transition from the state of absence of particles to the stationary state in which the scattering particles are present. The minima and maxima are then clearly seen on the data plot. This interference pattern, as in the case of the temperature dependence, is used to determine the rate of growth of cluster radius $r$ versus buffer gas pressure $p$. The results are shown in Fig. 5.

Again the rate of growth is almost constant and equal to $c a .0 .016 \mu \mathrm{m} / \mathrm{s}$, until the pressure reaches $c a$. $575 \mathrm{Tr}$. Then the rate of growth increases rapidly reaching the value of $0.038 \mu \mathrm{m} / \mathrm{s}$ for $p=700 \mathrm{Tr}$. We would like to remark that the rate of growth does not seem to depend on the laser power. The temperature and the buffer gas pressure measurements were performed for the different light power, 


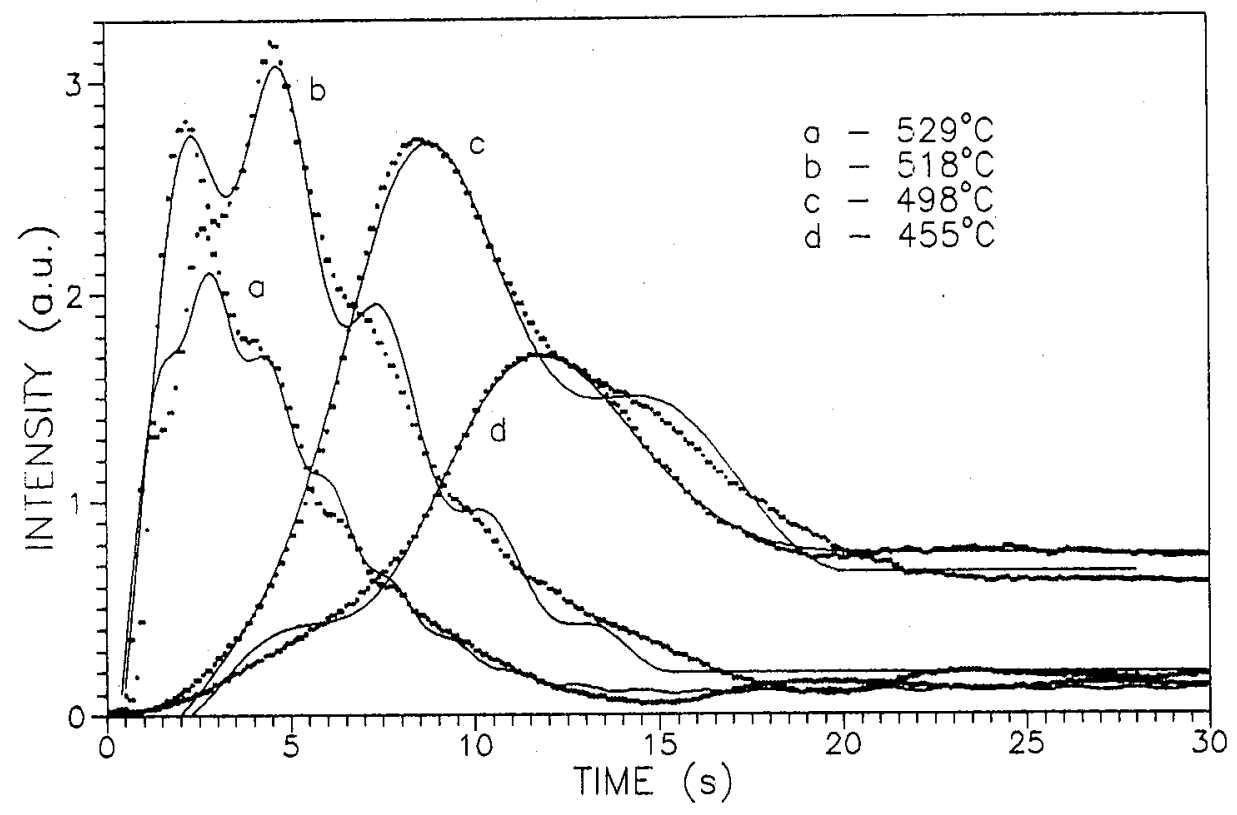

Fig. 2. The intensity of the $488 \mathrm{~nm}$ scattered light versus time for the first $30 \mathrm{~s}$ of the condensation process commenced by switching on the laser light at $t=0$. Plots for several temperatures. The solid line represents numerical fit of Eq. (6).

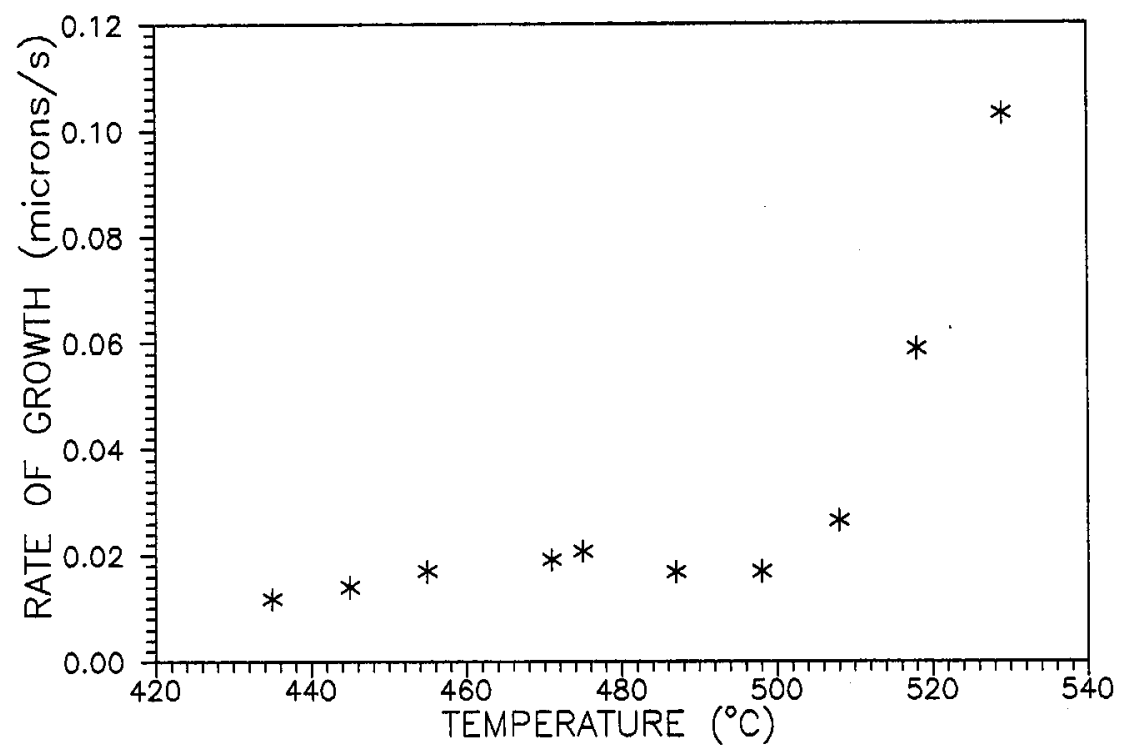

Fig. 3. The dependence of the rate of growth of clusters radius on the temperature. 

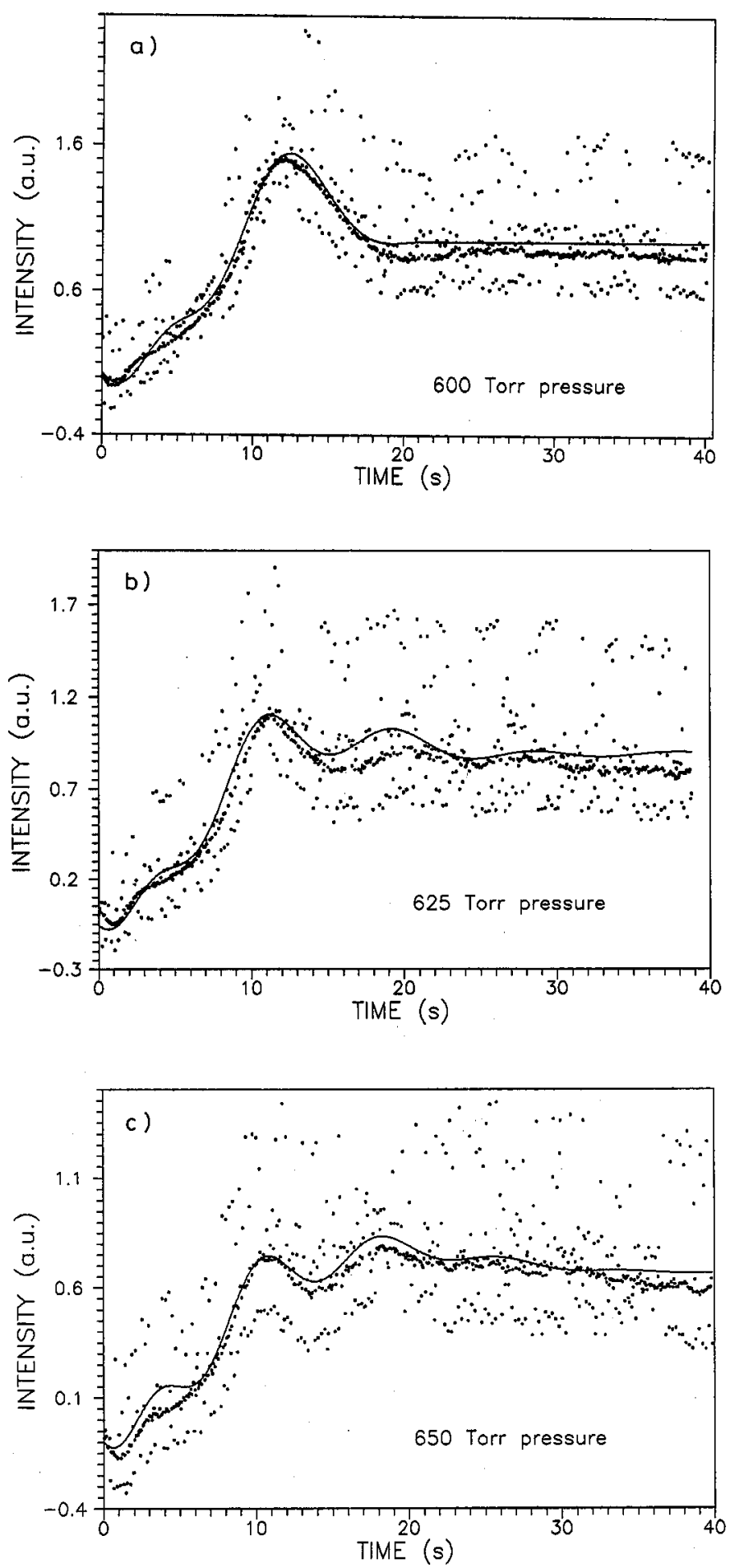

Fig. 4. The evolution of scattered light intensity for several values of buffer gas pressure. The solid line represents numerical fit of Eq. (6). 


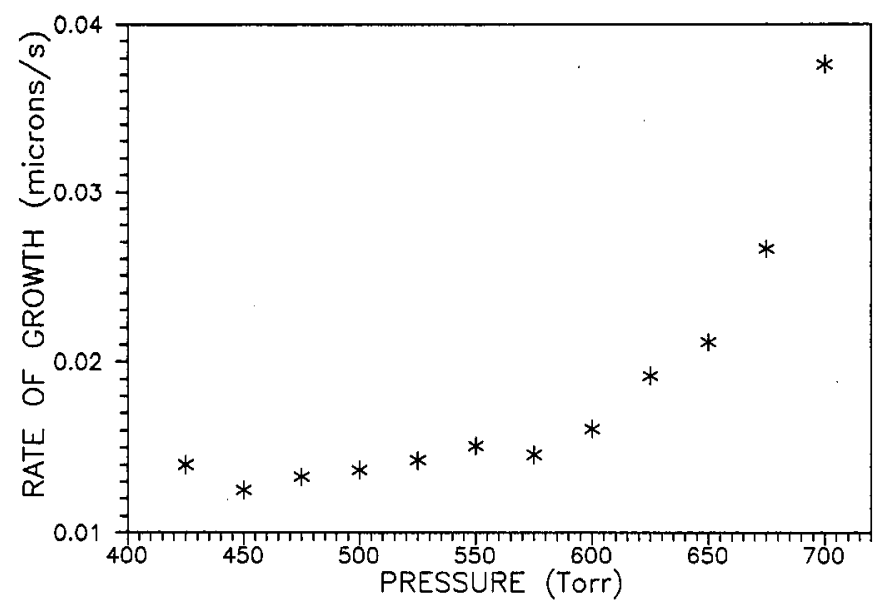

Fig. 5. The dependence of the rate of growth of clusters radius on the buffer gas pressure.

nevertheless for the low values of these parameters (temperature and pressure) $v$ has the same value.

\section{Discussion}

In this paper we have presented the experimental investigations of the laser light induced sodium clusters evolution as function of the buffer gas pressure $p$ and the temperature of the cell $T$. Following the analysis of the time-dependent intensity of the scattered light we can conclude that the cluster size is well-defined and grows linearly in time until it reaches final, stationary value. The model which was presented made possible to determine the rate of growth of sodium clusters. It was found to vary from 0.012 to $0.1 \mu \mathrm{m} / \mathrm{s}$ as the function of the pressure $p$ and the temperature $T$. Although our model of scattering and deconvolution of the single-particle scattering function from the number of clusters is very rough we think that our results are relatively precise.

\section{References}

[1] W.D. Knight, Z. Phys. D 12, 315 (1989).

[2] W.D. Knight, K. Clemenger, W.A. de Ileer, W.A. Saunders, M.Y. Chou, M.L. Cohen, Phys. Rev. Lett. 52, 2141 (1984).

[3] S. Giorgio, J. Urban, Z. Phys. D 12, 115 (1989).

[4] C. Brechignac, Ph. Cahuzac, J. Leygnier, R. Pflaum, J.Ph. Roux, J. Weiner, Z. Phys. D 12, 199 (1989).

[5] M. Mitome, Y. Tanichro, K. Takayanagi, Z. Phys. D 12, 45 (1989).

[6] R. Mosseri, J.F. Sadoc, Z. Phys. D 12, 89 (1989). 
[7] G.S. Anagnostatos, Phys. Lett. A 142, 146 (1989).

[8] G. Franke, J. Schulte, Z. Phys. D 12, 65 (1989).

[9] R.S. Berry, Z. Phys. D 12, 161 (1989).

[10] W.A. de Heer, W.D. Knight, M.Y. Chou, M.L. Cohen, Solid State Phys. 40, 93 (1987).

[11] J. Kowalski, T. Stehlin, F. Trager, M. Vollmer, Phase Transit. 24-26, 737 (1990).

[12] Physics and Chemistry of Small Clusters, Ed. P. Jena, B.K. Rao, S.N. Khanna, Plenum, New York 1987.

[13] S. Arvati, L.F. Dona Dalle Rose, P.L. Silvestrelli, F. Toigo, Nuovo Cimento D 11, 1063 (1989).

[14] W.D. Knight, W.A. de Heer, K. Clemenger, W.A. Saunders, Solid State Commun. 53, 445 (1985).

[15] M.M. Kappes, R.W. Kunz, E. Schumacher, Chem. Phys. Lett. 91, 413 (1982).

[16] D.M. Mann, H.P. Broida, J. Appl. Phys. 44, 4950 (1973).

[17] M. Allegrini, P. Bicchi, D. Dattrino, L. Moi, Opt. Commun. 49, 29 (1984).

[18] C.J. Duthler, S.E. Johnson, H.P. Broida, Phys. Rev. Lett. 26, 1236 (1971).

[19] W.A. de Heer, K. Selby, V. Kresin, J. Masui, M. Vollmer, A. Chatelain, W.D. Knight, Phys. Rev. Lett. 59, 1805 (1987).

[20] K. Selby, M. Vollmer, J. Masui, V. Kresin, W.A. de Heer, W.D. Knight, Phys. Rev. B 40, 5417 (1989).

[21] J.R. Heath, Phys. Rev. B 40, 9982 (1989).

[22] A.V. Butenko, P.A. Chubakov, Yu.E. Danikova, S.U. Karpov, A.K. Popov, S.G. Rautian, V.P. Safonov, V.U. Slabko, V.M. Shalaev, M.I. Stockman, Z. Phys. D 17, 283 (1990).

[23] W. Demtroder, M. McClintock, R.N. Zare, J. Chem. Phys. 51, 5495 (1969).

[24] M. Born, E. Wolf, Principles of Optics, Pergamon, Oxford 1965.

[25] H.C. van de Hulst, Light Scaltering by Small Particles, Wiley, New York 1957.

[26] K. Huang, Statistical Mechanics, Wiley, New York 1963. 